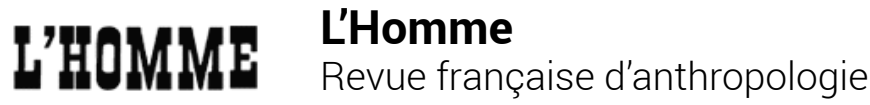

177-178 | 2006

Chanter, musiquer, écouter

\section{Göran Aijmer, New Year Celebrations in Central China in Late Imperial Times}

Hong Kong, The Chinese University Press, 2003, 188 p.

\section{Catherine Capdeville-Zeng}

\section{(2) OpenEdition}

\section{Journals}

Édition électronique

URL : http://journals.openedition.org/lhomme/2311

DOI : 10.4000//homme.2311

ISSN : 1953-8103

Éditeur

Éditions de l'EHESS

Édition imprimée

Date de publication : 1 juin 2006

Pagination : 549-550

ISSN : 0439-4216

Référence électronique

Catherine Capdeville-Zeng, "Göran Aijmer, New Year Celebrations in Central China in Late Imperial Times », L'Homme [En ligne], 177-178 | 2006, mis en ligne le 12 avril 2006, consulté le 24 septembre 2020. URL : http://journals.openedition.org/lhomme/2311 ; DOI : https://doi.org/10.4000/lhomme.2311

Ce document a été généré automatiquement le 24 septembre 2020

(C) École des hautes études en sciences sociales 


\title{
Göran Aijmer, New Year Celebrations in Central China in Late Imperial Times
}

Hong Kong, The Chinese University Press, 2003, 188 p.

\author{
Catherine Capdeville-Zeng
}

1 CE LIVRE est un essai d'anthropologie symbolique qui concerne les différentes célébrations du Nouvel An chinois dans le cours moyen du fleuve Yangzi, aux abords du lac Dongting. Cette étude est réalisée à partir de registres historiques, les "gazettes locales». Rédigées depuis le VII siècle, elles n'ont cessé d'être compilées par les mandarins des différentes dynasties pour documenter les coutumes locales, conserver les traces du passé et servir ainsi de référence morale. Malgré leur caractère orienté, l'auteur pense que « les lettrés chinois étaient en général de bons ethnographes en qui l'on peut avoir toute confiance »(p. 8). Néanmoins, ces gazettes étant régulièrement recopiées et réécrites, l'incertitude demeure souvent quant à la datation exacte de leurs informations. L'auteur a également étudié une chronique historique de la dynastie des Liang (502-536) ainsi que l'Encyclopédie impériale éditée au xvIII siècle. Il introduit enfin quelques éléments issus d'études contemporaines portant sur différentes régions de Chine. Le lecteur doit donc considérer que les «temps impériaux anciens » traités dans ce livre courent sur une période allant du $\mathrm{VI}^{\mathrm{e}}$ siècle jusqu'à l'époque contemporaine.

2 Le livre est organisé en deux parties, la première consacrée aux informations ethnographiques, et la seconde, plus courte, à leur analyse. Les chapitres sont concis et décrivent clairement les pratiques attachées à chacune des manifestations du Nouvel An. Ces festivités sont fixées d'après le cadre général du calendrier lunaire, mais incluent aussi deux fêtes associées au calendrier solaire.

3 La fête du La (le $8^{\mathrm{e}}$ jour $\mathrm{du} 12^{\mathrm{e}}$ mois), autrefois la fête la plus importante et supplantée aujourd'hui par le Nouvel An, se caractérise par une distribution de bouillie de riz. L'auteur interprète ce rite comme une distribution de la "force agnatique " pour " produire des enfants ». Cette offrande à Bouddha est aussi un cadeau pour nourrir les démons rôdeurs et affamés afin d'annihiler leur potentiel de destruction. 
4 Le solstice d'hiver (21 décembre) se distingue par des échanges de nouilles de riz, également symboles de la continuité agnatique. Le Petit Nouvel An (le $24^{\mathrm{e}}$ jour $\mathrm{du} 12^{\mathrm{e}}$ mois) commence par des exorcismes dansés pour éloigner les esprits négatifs. Ensuite, le dieu du Foyer est honoré dans la cuisine avec des offrandes, des lanternes et une réunion commensale. Après quoi, sa représentation en papier est brûlée et envoyée au ciel pour rapporter à l'empereur de Jade la conduite du groupe domestique pendant l'année écoulée. L'auteur interprète le dieu du Foyer comme « l'incarnation symbolique et sexuelle du groupe bilatéral » (p.120) et son renvoi au ciel par le besoin « d'éviter un clash avec les images d'agnation pure » (p. 120) qui vont devenir omniprésentes avec la visite prochaine des ancêtres (agnatiques). L'autre fait marquant est le balayage/ nettoyage des maisons pour leur souhaiter la bienvenue. Ces épisodes étaient sûrement des «tropes symboliques pour les forces polluantes des femmes mariées dans le lignage »(p. 121).

5 Le Nouvel An ( $1^{\mathrm{er}}$ jour du $1^{\mathrm{er}}$ mois) est d'abord fêté par une veillée à laquelle participent tous les membres d'une unité domestique, y compris les ancêtres agnatiques et les femmes, marquant ainsi un événement liminal, une « anti-structure commensale » (cf. Victor Turner). Mais dès le lendemain vient le temps de la réaffirmation des hiérarchies (surtout celle entre aînés et cadets) et des échanges de cadeaux entre parents - incluant les affins - sont effectués pendant plusieurs jours. Différents rites secondaires s'ajoutent ensuite.

6 Lors de la fête des Lanternes ( $15^{\mathrm{e}}$ jour du $1^{\mathrm{er}}$ mois) à la pleine lune, des lanternes sont exposées à l'extérieur pour indiquer aux ancêtres le chemin du retour dans le monde de l'obscurité. Les danses du dragon avaient la même fonction, et les processions de femmes étaient entendues comme des moyens d'écarter les maladies. Enfin, différentes sortes de représentations théâtrales avaient lieu et des offrandes étaient offertes à la Fille Violette, patronne de la soie.

7 Quant à l'Arrivée du printemps (le 5 février), cette fête marque le retour des activités agricoles signifiées par la destruction de la figurine en terre d'un bœuf et par un labour rituel.

8 L'auteur cherche à comprendre le sens symbolique de toutes ces activités rituelles, leur sens « iconique » ne relevant pas directement du langage ou des textes. Pour cela, il fait appel à ses études antérieures sans toujours démontrer ici ses analyses (comme par exemple le lien qu'il établit entre riz et force agnatique) et à des connaissances générales en sinologie. Il procède aussi à quelques comparaisons, dont les plus intéressantes mettent au jour des parallèles avec certaines autres fêtes annuelles : par exemple le balayage des maisons lors du Petit Nouvel An est comparé à celui des tombes lors de la fête des morts. Cela lui permet d'établir un tableau des rapports entre les différentes fêtes (p.161) et de démontrer de façon convaincante que le Nouvel An s'inscrit dans un cadre plus large, celui du temps social cyclique annuel.

9 L'auteur s'interroge aussi sur le sens à donner à l'existence des deux fêtes principales du Nouvel An. Le Petit Nouvel An marque la reproduction sociale à travers la consommation de riz qui transforme les femmes en sœurs et les enfants en descendants, à la différence du Nouvel An qui marque la reproduction sexuelle du groupe par l'incorporation des femmes étrangères à l'intérieur d'une structure bilatérale. Ainsi, « pendant le "réel" Nouvel An, l'affinité est centrale à la célébration de la domesticité » (p. 77). Enfin, la fête des Lanternes peut se comprendre comme la fête 
des Femmes (p.162) et il est significatif qu'elle se produise lors du départ des ancêtres agnatiques.

L'auteur a le mérite de chercher à replacer l'affinité dans la société chinoise alors qu'elle en a été presque totalement évacuée, notamment dans les analyses entreprises par Maurice Freedman et ses disciples. Cette place des affins semble malgré tout rester quelque peu secondaire dans les fêtes du Nouvel An. Enfin, que dire sur cette analyse "symbolique» sinon qu'elle laisse de côté des faits essentiels de structure. Par exemple, l'auteur s'interroge sur l'existence de « deux » Nouvel An, mais n'envisage pas de considérer la Fête des Lanternes comme un "troisième" Nouvel An. Une étude approfondie des termes employés montrerait pourtant facilement les liens entre ces trois fêtes du Nouvel An formant ensemble une structure rituelle simple : l'introduction avec le Petit Nouvel An, l'action principale avec le Nouvel An, et la conclusion avec la fête des Lanternes, également appelée dans d'autres régions le " (Nouvel) An Montant » (shangnian).

11 En conclusion, l'entreprise difficile de traiter de données historiques avec une méthode anthropologique est bien menée par l'auteur. Ce livre présente néanmoins quelques défauts. Il s'adresse plutôt au lecteur sinologue qu'au lecteur anthropologue. On regrette qu'il ne comporte pas de carte. Les mots chinois sont présentés différemment selon les occurrences et ne sont pas toujours expliqués (quelle différence entre les offrandes si et gong?), aucune règle claire concernant les transcriptions en caractères et les traductions n'apparaît. Il n'y a pas de glossaire et l'index rapporte uniquement les termes anglais. Mais encore, un tableau récapitulatif des chronologies ainsi qu'une présentation de la situation chinoise à propos de ses calendriers auraient aidé le lecteur à s'y retrouver. Ces imperfections n'enlèvent rien au caractère fascinant des éléments rapportés dans ce livre qui donnent matière à nourrir une réflexion autant anthropologique que sinologique. 